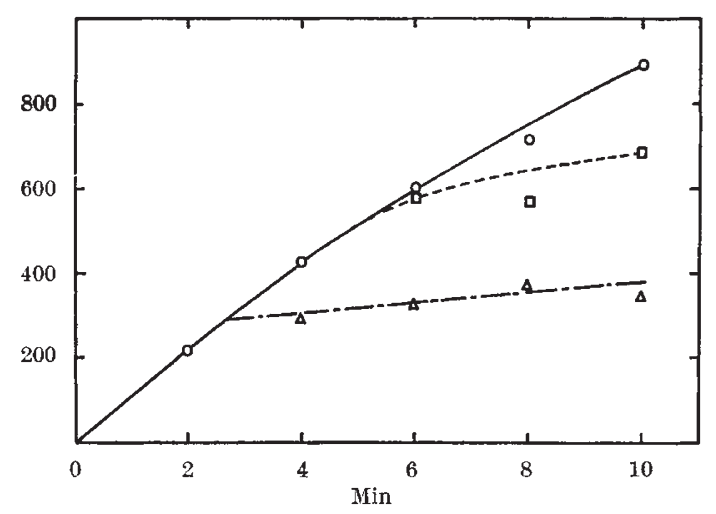

Fig. 2. The time course of RNA synthesis after addition of streptolydigin or rifampicin. Abscissa is the duration of the reaction and the ordinate is counts per min per $0.10 \mathrm{ml}$. reaction mix. The enzyme was "core" $\alpha_{2} \beta \beta^{\prime}$ and the assay conditions were as in Fig. $1.0-O$, Normal reaction $\square-\cdots \square$, rifampicin added at $2 \mathrm{~min}$ to a concentration of $2 \mu \mathrm{g} / \mathrm{ml}$. $\triangle--\Delta$, streptolydigin added at $2.5 \mathrm{~min}$ to a concentration of $10-\mathrm{M}$ rifampicin to which polymerase was added at $1 \mathrm{~min}$.

four did not change the inhibition curve from that shown in Fig. 1. This suggests that the drug and the triphosphates are not competing for the same site. It remains to be shown which subunits are altered in the mutants resistant to rifampicin, streptovaricin and streptolydigin.

I thank Bernard Weisblum for a preprint of his work with streptolydigin, and Andrew Travers, Dick Burgess, Chris Goff, Ned Minkley, James Watson and Walter Gilbert for their help.

The Biological Laboratories,

Robert SCHLEIF

Harvard University,

Cambridge, Massachusetts 02138.

Received July 14, 1969.

${ }^{1}$ Travers, A. A., and Burgess, R. R., Nature, 222, 537 (1969).

2 diMauro, E., Snyder, L., Marino, P., Lamberti, A., Coppo, A., and TocchiniValentini, G. P., Nature, 222, 533 (1969).

${ }^{3}$ Yura, T., and Igarashi, K., Proc. US Nat. Acad. Sci., 61, 1313 (1968)

${ }^{4}$ Siddhikol, C., Erbstoeszer, J., and Weisblum, B., J. Bact. (in the press).

5 Jacob, F., and Wollman, E. L., Sexuality and Genetics of Bacteria, 60 (Academic Press, New York, 1961).

${ }^{6}$ Adelberg, E. A., Mandel, M., and Chen, G. C. C., Biochem. Biophys. Res. Commun., 18, $788(1965)$.

'Mizano, S., Yamazaki, H., Nitta, K., and Umezawa, H., Biochem. Biophys. Res. Commun., 30, 379 (1968).

\section{Functional Independence of F and I Sex Pili}

ConJugation and gene transfer in $E$. coli and other Enterobacteria depend on the formation by donor cells of specialized filaments which may be the channel by which genes pass to the recipient ${ }^{1}$. These "scx pili" fall into two principal classes: "F pili" determined by the $F$ sex factor ${ }^{1}$ and by related $F$-like sex factors ${ }^{2}$ found in $\mathrm{fit}$ drug resistance (R) factors and colicin (Col) factors $\mathrm{V}$ and $\mathrm{B}$; and "I pili" determined by the I sex factors of Colla-CA53, Collb-P9, ColEla and fi- R factors ${ }^{2,3}$. The two classes of sex pilus seem to be distinct in many respects ${ }^{2}$, and this lack of relationship is further emphasized by the failure of an intact sex factor of one class to complement a mutant factor of the alternative class in pilus synthesis.

Mutant sex factors can bo selected by exposing cultures of donor bacteria to donor-specific phaget. Such cultures, however, are phage-sensitive initially only if they carry a sex factor which is de-repressed in respect of pilus synthesis so that most cells form sex pili and adsorb the phage $e^{5}$. The factor may be de-repressed either in the wild type state, as is F, or following mutation ${ }^{6}$, like the de-repressed mutant $f i+\mathrm{R}$ factor, $\mathrm{R} l d r d-19$, which fails to form repressor although remaining sensitive to repression (A. Frydman and E. Meynell, unpublished results). Selection for phage-resistance might therefore yield two types of mutant factor: those defective in pilus synthesis $(p s a-)$ and those which are $p s a+$ but fail to form pili because pilus synthesis has again become repressed. Cis-trans tests for repressor synthesis require that the mutant sex factor can readily coexist stably with a second repressible sex factor in the same cell; that is, superinfection immunity ${ }^{2,7}$ is absent. This is feasible with $F$ and a $f i+R$ factor but not with any pair of $I$ sex factors so far examined, such as ColIa and ColIb (ref. 7). Phageselected clones are therefore denoted here by an asterisk until their identity is established (for example, $F^{*}$ ).

Thirteen independent $F^{*}$ mutants were isolated from E. coli (Flac), strain AB1353, by phage MS2. All are believed to be $p s a-$, for none repressed Rldrd-19. That is, wild type $F$ does not seem to have a latent repressor region. Forty-two $I^{*}$ mutants were selected by $I$ specific phage ${ }^{8}$. All were derived from de-repressed mutants of Fredericq's complex plasmid consisting of ColIb-P9 linked to the trp cys region of $E$. coli ${ }^{9}$. Twenty-six of the mutants were derived from IP9drd-5 col-trpt and sixteen from IP9drd-5 colIb + trp +. Although super. infection immunity prevented a direct test for repression by these I* mutants, they were examined indirectly, as follows. De-repression of an I sex factor is recognizable not only by an increase in conjugating ability but also by examining broth cultures of their hosts which have been shaken overnight, for these have a smaller optical density and greater colicin titres than those of the same strain carrying the wild type factor. The sixteen I* derivatives of IP9drd-5 colI $b+\operatorname{trp}+$ were examined in this way: however, they did not fall into two discrete groups corresponding to the de-repressed and wild type plasmids but formed a continuous series between these extremes.

Complementation in pilus synthesis was tested by introducing several unrelated de-repressed I factors (IP9drd-5, R144drd, R163drd, R538drd and R64drd) into strain AB1353 carrying each of the $\mathrm{F}^{*}$ mutants. Similarly, wild type Flac or Rldrd-16 was tested in strains M616 or CL194 carrying the $I^{*}$ mutants. In no case did complementation occur, as shown by the failure of the $\mathrm{F}^{*}$ or $\mathrm{I}^{*}$ strains to become sensitive to $F$ or I phage, respectively, when drops of phage stock were applied to overlays inoculated with the test strains.

The two types of sex pilus thus seem to be completely distinct by every criterion applied so far. Not only are they structurally dissimilar in their morphology, antigenic structure and phage receptors ${ }^{2,3,8}$, but they are also functionally unrelated, as shown by the lack of complementation reported here, the differing specificities of their repressors ${ }^{2}$, and by the independent transfer of their factors when both are present in the same host ${ }^{10}$.

\section{Gr. G. MeYNELI}

Guinness-Lister Research Unit,

Eva AUFreiter

Lister Institute of Preventive Medicine,

Chelsea Bridge Road,

London SW1.

Received June 4, 1969

${ }^{1}$ Brinton, C. C., Trans. NY Acad. Sci., 27, 1003 (1965).

${ }^{2}$ Lawn, A. M., Meynell, E., Meynell, G. G., and Datta, N., Nature, 216. 343 (1967); Meynell, E., Moynell, G. G., and Datta, N., Bact. Rev., 32 it55 (1968).

2 Meynell, G. G., and Lawn, A. M., Genet. Res., Camb., 9, 359 (1967).

${ }^{4}$ Cuzin, F., and Jacob, F., Ann. Inst. Pasteur, 112, 1 (1967).

${ }^{5}$ Meynell, K., and Datta, N., Nature, 20\%, 884 (1965).

' Mevnell, E., and Datta, N., Nature, 214, 885 (1967); Edwards, S., and Meynell, G. G., ibid., 219, 869 (1968).

"Meynell, G. G., Genet. Res., Camb., 13, 113 (1969).

8 Meynell, G. G., and Lawn, A. M., Nature, 217, 1184 (1968).

' Fredericq, P., Zentbl. Bakt. Parasitkde, Abt. 1, Orig., 196, 142 (1965).

${ }^{10}$ Romero, E., and Meynell, E., J. Bact., 97, 780 (1969). 